\title{
Design of observer-based controllers for LPV systems with unknown parameters
}

\author{
W.P.M.H. Heemels, J. Daafouz, G. Millerioux
}

\begin{abstract}
Output-based feedback control of LPV systems is an important problem, as in practice it is rarely the case that the full state variable is available for feedback. In this paper we consider this problem in the case of discrete-time LPV systems for which the parameters are not exactly known, but only available with a finite accuracy or affected by noise during their measurement. The controllers are obtained using a separate design of an observer and a state feedback and the interconnection is proven to stabilize the LPV system despite the mismatch between the true and available parameters. The approach allows to maximize the parameter uncertainty while still guaranteeing closed-loop stability. In addition, it is possible to make tradeoffs between the admissible level of mismatch on the one hand and the performance in terms of decay factors on the other. All the design conditions will be formulated in term of LMIs, which can be solved efficiently, as is also illustrated by a numerical example.
\end{abstract}

Index Terms - LPV systems, output feedback and observers, robust control, LMIs, separation principle

\section{INTRODUCTION}

Linear Parameter-Varying (LPV) systems have received considerable attention from the control community in recent years due to their applicability in many practical situations (see [1], [2], [3], [4], [5], [6] and references therein). Controllers that are designed on the basis of LPV system models have to satisfy two important properties, when they are implemented in practice:

- First of all, the controller needs to be output-based, as in practice it is rarely the case that the full state variable is available for feedback.

- Secondly, the controller must be robust with respect to some degree of mismatch between the available and the true parameters as the real parameters are not always known exactly, although this is often assumed in the LPV literature.

The goal of this paper is to design controllers for LPV systems that satisfy these two properties, which means that we would like to solve the output-based controller design problem for discrete-time LPV systems with not exactly known parameters. The problem that the scheduling parameters measurements are only known up to a given precision was mentioned for the first time in [3] in the case of continuous-time LPV systems. Unfortunately, the synthesis of robust dynamic output feedback controllers in [3] has to

Maurice Heemels is with Eindhoven University of Technology, Dept. of Mechanical Engineering, Control Systems Technology, Eindhoven, The Netherlands, M.Heemels@tue.nl. Jamal Daafouz and Gilles Millerioux are with Nancy University, Centre de Recherche en Automatique de Nancy, France, jamal.daafouz@ensem.inpl-nancy.fr, gilles.millerioux@esstin.uhp-nancy.fr. be performed by the solution of bilinear matrix inequalities. In [7], one considered dynamic output feedback control of continuous-time LPV systems, where only some of the parameters are measured and available for feedback. The derived conditions for the construction of the controllers, which depend only on the measured parameters, are expressed in terms of linear matrix inequalities (LMIs) and an additional coupling constraint, which destroys the convexity of the conditions. Recently, a solution is given in [8] to the robust dynamic output feedback design for continuous-time LPV systems when the measured varying parameters do not exactly fit the real ones using convex programming.

Output feedback control design for discrete-time LPV systems for which the measured parameters do not exactly fit the real ones is an open problem. Convexity is only obtained in case of stability analysis [9]. In [9] it is also shown that an observer that is asymptotically recovering the state when the parameters are exactly measured, is inputto-state stable (ISS) [10], [11] with respect to mismatch between the true and the available parameters. However, [9] does not study the observer synthesis nor the outputbased stabilization problem. In addition, it does not allow for minimizing the ISS gain (as a measure for the influence of the mismatch of the parameters on the estimation error). These two important features will be considered in this paper.

Closely related to LPV systems are switched linear (SL) systems, which can be perceived as a subclass of LPV systems in which the parameters only take a finite number of values. Observer-based control design for SL systems has been considered in [12] under the assumption of having exact knowledge of the parameter values. In case of unknown parameters, [13] proposes design conditions for observers that include an estimation procedure for the parameters. This procedure exploits that the number of parameter values is finite, which makes it not applicable to general LPV systems. As a consequence, output-based controller design for discrete-time LPV systems with unknown parameters is at present an open problem.

This paper contributes to this open problem. In particular, the main contributions are LMI-based conditions for the separate design of state observers and input-to-state stabilizing state feedbacks for discrete-time LPV systems in the situation when the parameters are not exactly known. Next we prove that the resulting closed-loop system is globally exponentially stable for some level of mismatch between the true parameters and the available ones. Interestingly, the flexibility in our framework allows to make tradeoffs between this level of mismatch and the performance of the closed- 
loop in terms of the decay factor. All the design conditions will be formulated in terms of LMIs, which can be solved efficiently [14]. This will be demonstrated for an example.

The paper is organized as follows. The next section is dedicated to notations and basic definitions used in the sequel. Section III gives the problem statement of observer-based control design for LPV systems with the assumption that the scheduling parameters used by the controller do not exactly match the real ones. In sections IV and V the observer and the state feedback are designed separately. Section VI shows that the separate design of the observer and the state feedback leads to stabilizing output-based controllers. Also a design procedure giving the largest level of uncertainty is proposed. We end the paper by a numerical example that illustrates the main features of the proposed approach and conclusions.

\section{NOTATION AND BASIC DEFINITIONS}

$\mathbb{R}, \mathbb{R}_{\geq 0}$, and $\mathbb{N}$ are the field of real numbers, the set of non-negative reals and the set of non-negative integers, respectively. The $i$-th entry of a real vector $x$ is denoted by $x^{i}$ (subscripts are used for denoting discrete-time dependence). We denote by $\|x\|=\sqrt{x^{T} x}$ the Euclidean norm of $x$ in $\mathbb{R}^{n}$, where $M^{T}$ denotes the transpose for a vector or matrix $M$, and by $\|x\|_{\infty}$ its infinity norm given by $\max _{i}\left|x^{i}\right|$. For a sequence $\left\{v_{k}\right\}_{k \in \mathbb{N}}$ with $v_{k} \in \mathbb{R}^{n}$ we denote its supremum norm $\sup _{k \in \mathbb{N}}\left\|v_{k}\right\|$ by $\|v\|_{\infty}$. For a matrix $M \in \mathbb{R}^{n \times m}$ we denote its spectral norm $\sqrt{\lambda_{\max }\left(M^{T} M\right)}$ by $\|M\|$, where $\lambda_{\max }\left(M^{T} M\right)$ denotes the largest eigenvalue of $M^{T} M$. When a matrix $P$ is positive definite (including symmetry), we write $P \succ 0$. If it is positive semi-definite, we use $P \succeq 0$. Similarly, for (semi-)negative definiteness we write $\prec$ and $\preceq$. By 0 and 1 we denote the zero and the identity matrix of appropriate dimensions.

A function $\varphi: \mathbb{R}_{+} \rightarrow \mathbb{R}_{+}$belongs to class $\mathcal{K}$ if it is continuous, strictly increasing and $\varphi(0)=0$ and to class $\mathcal{K}_{\infty}$ if additionally $\varphi(s) \rightarrow \infty$ as $s \rightarrow \infty$. A function $\beta$ : $\mathbb{R}_{+} \times \mathbb{R}_{+} \rightarrow \mathbb{R}_{+}$belongs to class $\mathcal{K} \mathcal{L}$ if for each fixed $k \in \mathbb{R}_{+}, \beta(\cdot, k) \in \mathcal{K}$ and for each fixed $s \in \mathbb{R}_{+}, \beta(s, \cdot)$ is decreasing and $\lim _{k \rightarrow \infty} \beta(s, k)=0$. Consider now the discrete-time nonlinear systems

$$
x_{k+1}=G\left(x_{k}, \omega_{k}\right),
$$

and

$$
x_{k+1}=G_{v}\left(x_{k}, v_{k}, \omega_{k}\right),
$$

where $x_{k} \in \mathbb{R}^{n}$ is the state, $v_{k} \in \mathbb{R}^{d_{v}}$ is an unknown disturbance input and $\omega_{k} \in \mathbb{R}^{d_{\omega}}$ is an uncertainty parameter at discrete time $k \in \mathbb{N}$. $G: \mathbb{R}^{n} \times \mathbb{R}^{d_{\omega}} \rightarrow \mathbb{R}^{n}$ and $G_{v}: \mathbb{R}^{n} \times \mathbb{R}^{d_{v}} \times \mathbb{R}^{d_{\omega}} \rightarrow \mathbb{R}^{n}$ are arbitrary nonlinear functions. We assume that $\omega_{k} \in \Omega, k \in \mathbb{N}$ for some set $\Omega \subset \mathbb{R}^{d_{\omega}}$. Next, we define the notions of global asymptotic stability (GAS) and global exponential stability (GES) for (1) and input-tostate stability (ISS) [10], [11] for (2).

Definition 1: [10], [11] The system (1) with uncertainty set $\Omega$ is called globally asymptotically stable (GAS), if there exists a $\mathcal{K} \mathcal{L}$-function $\beta$ such that, for each $x_{0} \in \mathbb{R}^{n}$ and all $\left\{\omega_{k}\right\}_{k \in \mathbb{N}}$ with $\omega_{k} \in \Omega, k \in \mathbb{N}$, it holds that the corresponding state trajectory satisfies

$$
\left\|x_{k}\right\| \leq \beta\left(\left\|x_{0}\right\|, k\right)
$$

for all $k \in \mathbb{N}$. If $\beta$ can be taken of the form $\beta(s, k)=d s \lambda^{k}$ for some $d \geq 0$ and $0 \leq \lambda<1$ the system (1) with uncertainty set $\Omega$ is called globally exponentially stable (GES). The system (2) with uncertainty set $\Omega$ is said to be input-to-state stable (ISS) with respect to $v$ if there exist a $\mathcal{K} \mathcal{L}$-function $\beta$ and a $\mathcal{K}$-function $\gamma$ such that, for each $x_{0} \in \mathbb{R}^{n}$, all $\left\{v_{k}\right\}_{k \in \mathbb{N}}$ and all $\left\{\omega_{k}\right\}_{k \in \mathbb{N}}$ with $\omega_{k} \in \Omega, k \in \mathbb{N}$, it holds for all $k \in \mathbb{N}$ that

$$
\left\|x_{k}\right\| \leq \beta\left(\left\|x_{0}\right\|, k\right)+\gamma\left(\|v\|_{\infty}\right) .
$$

We call $\lambda$ a decay factor for (1) and the function $\gamma$ an ISS gain of (2). Next we state sufficient conditions for ISS using so-called ISS Lyapunov functions. The proofs are omitted for shortness, but can be based on [11], [15] by including the uncertainty parameter and adopting parameter-dependent Lyapunov functions.

Theorem 2: Let $d_{1}, d_{2} \in \mathbb{R}_{\geq 0}$, let $a, b, c, \mu \in \mathbb{R}_{>0}$ with $c \leq b$ and let $\alpha_{1}(s):=a s^{\mu}, \alpha_{2}(s):=b s^{\mu}, \alpha_{3}(s):=c s^{\mu}$ and $\sigma \in \mathcal{K}$. Furthermore, let $V: \mathbb{R}^{n} \times \mathbb{R}^{d_{\omega}} \rightarrow \mathbb{R}_{\geq 0}$ be a function such that

$$
\begin{aligned}
\alpha_{1}(\|x\|) \leq V(x, \omega) & \leq \alpha_{2}(\|x\|) \\
V\left(G_{v}\left(x, v, \omega_{1}\right), \omega_{2}\right)-V\left(x, \omega_{1}\right) & \leq-\alpha_{3}(\|x\|)+\sigma(\|v\|)
\end{aligned}
$$

for all $x \in \mathbb{R}^{n}$, all $v \in \mathbb{R}^{d_{v}}$ and $\omega, \omega_{1}, \omega_{2} \in \Omega$. Then system (2) with uncertainty set $\Omega$ is ISS with respect to $v$. In case (4a) and $V\left(G\left(x, \omega_{1}\right), \omega_{2}\right)-V\left(x, \omega_{1}\right) \leq-\alpha_{3}(\|x\|)$ hold for all $x \in \mathbb{R}^{n}$ and $\omega, \omega_{1}, \omega_{2} \in \Omega$, then system (1) with uncertainty set $\Omega$ is GES with decay factor $1-\frac{c}{b} \in[0,1)$.

A function $V$ that satisfies (4) is called an ISS Lyapunov function.

\section{PRoblem STATEMENT}

We consider discrete-time linear parameter-varying (LPV) systems given by

$$
\begin{aligned}
x_{k+1} & =A\left(\rho_{k}\right) x_{k}+B u_{k} \\
y_{k} & =C x_{k}+D u_{k}
\end{aligned}
$$

with $x_{k} \in \mathbb{R}^{n}, y_{k} \in \mathbb{R}^{m}$ and $u_{k} \in \mathbb{R}^{r}$ the state, output and control input at discrete time $k \in \mathbb{N}$ and $\rho_{k} \in \mathbb{R}^{L}$ is a time-varying parameter. The matrices $A(\rho) \in \mathbb{R}^{n \times n}$ for each $\rho, B \in \mathbb{R}^{n \times r}, C \in \mathbb{R}^{m \times n}, D \in \mathbb{R}^{m \times r}$ have appropriate dimensions. The parameter $\rho$ lies in some set $\Theta \subset \mathbb{R}^{L}$ and we assume that $A: \Theta \rightarrow \mathbb{R}^{n \times n}$ can be written in the polytopic form

$$
A(\rho)=\sum_{i=1}^{N} \xi^{i}(\rho) A_{i}
$$

for certain continuous functions $\xi^{i}: \Theta \rightarrow \mathbb{R}$ and matrices $A_{i} \in \mathbb{R}^{n \times n}, i=1, \ldots, N$. In addition we assume that the mapping $\xi: \Theta \rightarrow \mathbb{R}^{N}$ given by $\xi:=\left(\xi^{1}, \ldots, \xi^{N}\right)^{\top}$ is 
such that $\xi(\Theta) \subset \mathcal{S}$ with $\mathcal{S}=\left\{\mu \in \mathbb{R}^{N} \mid \mu^{i} \geq 0, i=\right.$ $1, \ldots, N$ and $\left.\sum_{i=1}^{N} \mu^{i}=1\right\}$. Hence, $A(\rho)$ lies for each $\rho \in \Theta$ in the convex hull $\mathbf{C o}\left\{A_{1}, \ldots, A_{N}\right\}$.

In this paper, we focus on the situation where the true (time-varying) parameter $\rho_{k}$ is actually not available, but only an estimated parameter $\hat{\rho}_{k} \in \Theta$ fulfilling $\left\|\rho_{k}-\hat{\rho}_{k}\right\|_{\infty} \leq$ $\Delta$, where $\Delta$ is some nonnegative constant indicating the uncertainty level. The aim in this paper is to tackle the following design problem.

Problem 3: Design an observer-based controller of the form

$$
\begin{aligned}
\hat{x}_{k+1} & =A\left(\hat{\rho}_{k}\right) \hat{x}_{k}+B u_{k}+L\left(\hat{\rho}_{k}\right)\left(y_{k}-\hat{y}_{k}\right) \\
\hat{y}_{k} & =C \hat{x}_{k}+D u_{k} \\
u_{k} & =K\left(\hat{\rho}_{k}\right) \hat{x}_{k}
\end{aligned}
$$

with $L\left(\hat{\rho}_{k}\right)=\sum_{i=1}^{N} \xi^{i}\left(\hat{\rho}_{k}\right) L_{i}$ and $K\left(\hat{\rho}_{k}\right)=\sum_{i=1}^{N} \xi_{k}^{i}\left(\hat{\rho}_{k}\right) K_{i}$ by appropriately choosing the gains $L_{i}$ and $K_{i}, i=1, \ldots, N$ such that the closed-loop system (5)-(7) is GAS when the uncertainty satisfies $\left\|\rho_{k}-\hat{\rho}_{k}\right\|_{\infty} \leq \Delta$ and $\hat{\rho}_{k} \in \Theta$ for all $k \in \mathbb{N}$.

As a second goal we aim at designing an observerbased controller as in the above problem formulation that guarantees GAS of the closed-loop system for the largest uncertainty level $\Delta$.

The approach we will take is to design the observer and the state feedback separately and provide a formal proof that the interconnection stabilizes the LPV system (5) (see section VI).

\section{OBSERVER DESIGN}

We first focus on the estimation of the state $x_{k}$ using a so-called polytopic observer of the form, which is given by

$\left\{\begin{array}{l}\hat{x}_{k+1}=A\left(\hat{\rho}_{k}\right) \hat{x}_{k}+B u_{k}+L\left(\hat{\rho}_{k}\right)\left(y_{k}-\hat{y}_{k}\right) \\ \hat{y}_{k}=C \hat{x}_{k}+D u_{k},\end{array}\right.$

where $\hat{\rho}_{k} \in \Theta$ and possibly $\rho_{k} \neq \hat{\rho}_{k}$. The estimation error $e_{k}:=x_{k}-\hat{x}_{k}$ is governed by

$$
e_{k+1}=\mathcal{A}_{e}\left(\hat{\rho}_{k}\right) e_{k}+v_{k}
$$

with $\mathcal{A}_{e}\left(\rho_{k}\right):=\sum_{i=1}^{N} \xi^{i}\left(\rho_{k}\right) \tilde{A}_{i}$, where $\tilde{A}_{i}=A_{i}-L_{i} C$ and

$$
v_{k}=(\underbrace{A\left(\rho_{k}\right)-A\left(\hat{\rho}_{k}\right)}_{=: \Delta A\left(\rho_{k}, \hat{\rho}_{k}\right)}) x_{k}
$$

The next theorem provides polytopic observers (8) that render (9) ISS with respect to $v$.

Theorem 4: Assume that there exist symmetric matrices $P_{i} \in \mathbb{R}^{n \times n}$, matrices $G_{i} \in \mathbb{R}^{n \times n}, F_{i} \in \mathbb{R}^{n \times m}, i=1, \ldots, N$ and a scalar $\sigma_{e v}$ satisfying for all $i, j=1, \ldots, N$ the following LMIs

$$
\left[\begin{array}{cccc}
G_{i}^{T}+G_{i}-P_{j} & \mathbf{0} & G_{i} A_{i}-F_{i} C & G_{i} \\
\mathbf{0} & \mathbf{1} & \mathbf{1} & \mathbf{0} \\
A_{i}^{T} G_{i}^{T}-C^{T} F_{i}^{T} & \mathbf{1} & P_{i} & \mathbf{0} \\
G_{i}^{T} & \mathbf{0} & \mathbf{0} & \sigma_{e v} \mathbf{1}
\end{array}\right] \succ \mathbf{0}
$$

then the error dynamics (9) with uncertainty set $\Theta$ for $\hat{\rho}$ and $^{1} L_{i}=G_{i}^{-1} F_{i}$ is ISS with respect to $v$ and $V_{e}\left(e_{k}, \hat{\xi}_{k}\right)=$ $e_{k}^{T}\left(\sum_{i=1}^{N} \hat{\xi}_{k}^{i} P_{i}\right) e_{k}$ is an ISS Lyapunov function that satisfies

$$
\begin{array}{r}
V_{e}\left(e_{k+1}, \hat{\xi}_{k+1}\right)-V_{e}\left(e_{k}, \hat{\xi}_{k}\right) \leq-\left\|e_{k}\right\|^{2}+\sigma_{e v}\left\|v_{k}\right\|^{2} \\
\left\|e_{k}\right\|^{2} \leq V_{e}\left(e_{k}, \hat{\xi}_{k}\right) \leq \sigma_{e v}\left\|e_{k}\right\|^{2}
\end{array}
$$

for all $\hat{\xi}_{k}, \hat{\xi}_{k+1} \in \mathcal{S}, e_{k} \in \mathbb{R}^{n}, v_{k} \in \mathbb{R}^{n}$. The ISS gain $\gamma$ can be taken linear as $\gamma(s)=\sigma_{e v} s$.

Proof: The proof can be found in [16].

In case the conditions of Theorem 4 hold, the polytopic observer (8) guarantees GES of the error dynamics (9) in the nominal case where $\rho_{k}=\hat{\rho}_{k}$ for all $k \in \mathbb{N}$ (as then $v_{k}=0$, $k \in \mathbb{N}$ ). In case $\rho_{k} \neq \hat{\rho}_{k}$, ISS guarantees only a steady state estimation error $e$ that is smaller than $\delta \sigma_{e v} \sup _{k \in \mathbb{N}}\left\|x_{k}\right\|$ with $\delta:=\sup \left\{\Delta A(\rho, \hat{\rho}) \mid\|\rho-\hat{\rho}\|_{\infty} \leq \Delta\right\}$ (as the ISS gain is $\left.\gamma(s)=\sigma_{e v} s\right)$. Hence, a kind of steady state relative error can be obtained in the sense that

$$
\frac{\limsup _{k \rightarrow \infty}\left\|e_{k}\right\|}{\lim \sup _{k \rightarrow \infty}\left\|x_{k}\right\|} \leq \delta \sigma_{e v},
$$

which implies that $e_{k} \rightarrow 0(k \rightarrow \infty)$, if $\left(\rho_{k}-\hat{\rho}_{k}\right) \rightarrow 0$ $(k \rightarrow \infty)$.

Remark 5: In [17] also the concept of steady state relative error was used in the context of observer design for discontinuous PWA systems in which the mode of the plant can be different than the mode of the observer. Here this mismatch between observer and plant model is caused by $\rho_{k} \neq \hat{\rho}_{k}$, which has a similar effect as in [17].

The smallest ISS gain $\sigma_{e v}$ based on the above design procedure can be obtained by selecting among all possible solutions $\sigma_{e v}, P_{i}, G_{i}$, and $F_{i}$ of the LMIs (11) for $i, j=$ $1, \ldots, N$ the ones leading to the smallest value for $\sigma_{e v}$, which amounts to solving the convex optimization problem

$$
\min \left\{\sigma_{e v} \mid P_{i}, F_{i}, G_{i}, \sigma_{e v} \text { satisfying }(11) i, j=1, \ldots, N\right\}
$$

Remark 6: Note that the normalization of certain constants in (12) to 1 is without loss of generality as any ISS Lyapunov function $V_{e}$ for (9) can be multiplied by a sufficiently large positive constant to satisfy (12). See also the proof of Theorem 7 below.

As mentioned, if the hypotheses of Theorem 4 are satisfied, the polytopic observer (8) guarantees GES of the error dynamics in the nominal case $\left(\rho_{k}=\hat{\rho}_{k}\right.$ for all $\left.k \in \mathbb{N}\right)$. In other words, the observer (8) asymptotically recovers the state of the LPV system when the parameters $\rho_{k}$ (and thus $\left.\xi_{k}\right)$ are exactly known. Actually, under the hypotheses of Theorem 4, the observer satisfies the matrix inequalities

$$
\begin{aligned}
0 & \succ \quad\left(A_{i}-L_{i} C\right)^{T} \tilde{P}_{j}\left(A_{i}-L_{i} C\right)-\tilde{P}_{i}, \\
& i, j=1, \ldots, N \\
0 & \prec \quad \tilde{P}_{i}, i=1, \ldots, N
\end{aligned}
$$

\footnotetext{
${ }^{1}$ The LMIs (11) imply that $G_{i}$ is invertible for each $i=1, \ldots, N$ as is shown in the proof.
} 
In [9] it is proven that (15) is sufficient for the observer (8) with $\hat{\rho}_{k}=\rho_{k}$ (a nominal observer) to recover the state of (5) asymptotically. Clearly, for an observer to work also in case $\rho_{k} \neq \hat{\rho}_{k}$ the inequalities in (15) are necessary conditions (provided one adopts parameter-dependent quadratic Lyapunov functions). Interestingly, the conditions in (15) also guarantee that the hypotheses of Theorem 4 are satisfied (as will be shown in Theorem 7 below). This shows the non-conservatism of the LMIs (11) as the existence of a nominal observer for the exact LPV system, with a parameter-dependent quadratic Lyapunov function proving GES of the error dynamics, is sufficient for (11) to hold. This also shows that any GES observer for the exact LPV system has some degree of robustness in the sense that it has some steady state relative error as in (13) for some $\sigma_{e v}>0$.

Theorem 7: If there exist $\tilde{P}_{i}$ and $L_{i}, i=1, \ldots, N$ such that (15) holds, then there are symmetric matrices $\tilde{P}_{i}$ and matrices $F_{i}, G_{i}, i=1, \ldots, N$ and a scalar $\sigma_{e v}$ satisfying for all $i, j=1, \ldots, N$ the LMIs (11).

Proof: The proof can be found in [16].

\section{STATE FEEDBACK DESIGN}

We now focus on the design of a state feedback for (5a) using an estimated state given by

$$
u_{k}=K\left(\hat{\rho}_{k}\right) \hat{x}_{k}=K\left(\hat{\rho}_{k}\right)\left(x_{k}-e_{k}\right)
$$

with $K\left(\hat{\rho}_{k}\right)=\sum_{i=1}^{N} \xi^{i}\left(\hat{\rho}_{k}\right) K_{i}$ and $e_{k}$ the estimation error. This results in the closed loop

$$
x_{k+1}=\mathcal{A}_{x}\left(\hat{\rho}_{k}\right) x_{k}+v_{k}-B K\left(\hat{\rho}_{k}\right) e_{k}
$$

with, as before, $v_{k}$ is given by (10) and $\mathcal{A}_{x}\left(\hat{\rho}_{k}\right)=$ $\sum_{i=1}^{N} \xi^{i}\left(\hat{\rho}_{k}\right) \underbrace{\left(A_{i}+B K_{i}\right)}_{A_{B K_{i}}}$. Again, we sometimes write $\hat{\xi}_{k}^{i}=$ $\xi^{i}\left(\hat{\rho}_{k}\right)$ and $\xi_{k}^{i}=\xi^{i}\left(\rho_{k}\right)$. We now study ISS of (17).

Theorem 8: Assume that there exist symmetric matrices $Y_{i} \in \mathbb{R}^{n \times n}$ and matrices $Z_{i} \in \mathbb{R}^{m \times n}, i=1, \ldots, N$ and scalars $\sigma_{x v}, \sigma_{x e}, \mu$ with $\mu>0$ satisfying for $i, j=1, \ldots, N$ the LMI conditions

$$
\left[\begin{array}{ccccc}
Y_{i} & \mathbf{0} & \mathbf{0} & Y_{i} A_{i}^{T}+Z_{i}^{T} B^{T} & Y_{i} \\
\mathbf{0} & \sigma_{x v} \mathbf{1} & \mathbf{0} & \mathbf{1} & \mathbf{0} \\
\mathbf{0} & \mathbf{0} & \sigma_{x e} \mathbf{1} & -Z_{i}^{T} B & \mathbf{0} \\
A_{i} Y_{i}+B Z_{i} & \mathbf{1} & -B Z_{i} & Y_{j} & \mathbf{0} \\
Y_{i} & \mathbf{0} & \mathbf{0} & \mathbf{0} & \mathbf{1}
\end{array}\right] \succ \mathbf{0}
$$

and for $i=1, \ldots, N$

$$
Y_{i} \succeq \mu \mathbf{1},
$$

then the closed-loop system (17) with uncertainty set $\Theta$ for $\hat{\rho}$ and $K_{i}=Z_{i} Y_{i}^{-1}, i=1, \ldots, N$ is ISS with respect to $e$ and $v$ and $V_{x}\left(x_{k}, \hat{\xi}_{k}\right)=x_{k}^{T} \sum_{i=1}^{N} \hat{\xi}_{k}^{i} S_{i} x_{k}$ is an ISS Lyapunov function that satisfies for all $\xi_{k}, \hat{\xi}_{k+1} \in \mathcal{S}$, all $x_{k} \in \mathbb{R}^{n}$, all $e_{k} \in \mathbb{R}^{n}$ and all $v_{k} \in \mathbb{R}^{n}$

$$
\begin{gathered}
V_{x}\left(x_{k+1}, \hat{\xi}_{k+1}\right)-V_{x}\left(x_{k}, \hat{\xi}_{k}\right) \leq \\
\leq-\left\|x_{k}\right\|^{2}+\sigma_{x v}\left\|v_{k}\right\|^{2}+\mu^{-2} \sigma_{x e}\left\|e_{k}\right\|^{2}, \\
\left\|x_{k}\right\|^{2} \leq V_{x}\left(x_{k}, \hat{\xi}_{k}\right) \leq \sigma_{x v}\left\|x_{k}\right\|^{2} .
\end{gathered}
$$

Proof: The proof is given in [16].

The following corollary can be obtained immediately from the above theorem in case the full state $x_{k}$ is known (i.e. $e_{k}=$ 0 for all $k \in \mathbb{N}$ ) and thus we consider the state feedback law

$$
u_{k}=K\left(\hat{\rho}_{k}\right) x_{k} .
$$

Corollary 9: Let the hypotheses of Theorem 8 be satisfied. Then the LPV system consisting of (5a) and the state feedback (20) with uncertainty set $\Theta$ for $\rho$ and $K_{i}=$ $Z_{i} Y_{i}^{-1}, i=1, \ldots, N$ is GES for all uncertainties satisfying $\|\Delta A(\rho, \hat{\rho})\| \leq \delta$, when $\delta<\frac{1}{\sigma_{x v}}$.

Proof: From (19a) with $e=0$ and $v_{k}=\Delta A\left(\rho_{k}, \hat{\rho}_{k}\right) x_{k}$ it follows that

$$
V_{x}\left(x_{k+1}, \hat{\xi}_{k+1}\right)-V_{x}\left(x_{k}, \hat{\xi}_{k}\right) \leq-\left(1-\sigma_{x v} \delta\right)\left\|x_{k}\right\|^{2} .
$$

Together with (19b) this proves GES on the basis of Theorem 2.

An analogous result to Theorem 7 can also be shown for the state feedback. In particular, a nominal state feedback

$$
u_{k}=K\left(\rho_{k}\right) x_{k}
$$

with $K\left(\rho_{k}\right)=\sum_{i=1}^{N} \xi^{i}\left(\rho_{k}\right) K_{i}$ (i.e. with estimation error $e_{k}=0, k \in \mathbb{N}$ and exact knowledge of parameters, $\rho_{k}=\hat{\rho}_{k}$, $k \in \mathbb{N}$ ) coupled to the LPV system (5a) is GAS if there are $K_{i}, \tilde{S}_{i}, i=1, \ldots, N$ such that

$$
\begin{gathered}
0 \succ\left(A_{i}+B K_{i}\right)^{T} \tilde{S}_{j}\left(A_{i}+B K_{i}\right)-\tilde{S}_{i}, i, j=1, \ldots, N \\
\quad \text { and } 0 \prec S_{i}, i=1, \ldots, N .
\end{gathered}
$$

Clearly, a state feedback (16) that renders (17) ISS (proved by parameter-dependent quadratic ISS Lyapunov functions) certainly satisfies (23). Interestingly, the converse also holds in the sense that a nominally stabilizing state feedback for (5a) has some robustness properties in the sense that (19) holds for some $V_{x}$ and even stronger, the LMIs in (18) are feasible. This clearly indicates the non-conservatism of the derived LMIs in Theorem 8. However, note that (23) does not allow any minimization of the ISS gains, while the results of Theorem 8 do.

Theorem 10: Suppose that there exist $K_{i}, \tilde{S}_{i}, i=$ $1, \ldots, N$ such that (23) is satisfied. Then there are symmetric matrices $Y_{i}$ and matrices $Z_{i}, i=1, \ldots, N$ and scalars $\sigma_{x v}, \sigma_{x e}, \mu$ with $\mu>0$ satisfying the LMIs (18) for $i, j=$ $1, \ldots, N$.

Proof: The reader is referred to [16] for the proof.

\section{OBSERVER-BASED CONTROL DESIGN}

Next we will show that the separate design of the observer as in section IV and a state feedback as in section V leads to a stabilizing output-based controller for some nontrivial level of uncertainty $\delta:=\sup \left\{\|\Delta A(\rho, \hat{\rho})\| \mid\|\rho-\hat{\rho}\|_{\infty} \leq\right.$ $\Delta\}$. Actually, in the end we aim at synthesizing outputbased controllers that stabilize the closed-loop system (5), (7) for the largest level of uncertainty $\delta$ by using convex programming techniques.

Assume that the observer has been designed according to section IV and the state feedback according to section V. The closed-loop system is given by 


$$
\left(\begin{array}{c}
x_{k+1} \\
e_{k+1}
\end{array}\right)=\left[\begin{array}{cc}
A\left(\rho_{k}\right)+B K\left(\hat{\rho}_{k}\right) & -B K\left(\hat{\rho}_{k}\right) \\
A\left(\rho_{k}\right)-A\left(\hat{\rho}_{k}\right) & A\left(\hat{\rho}_{k}\right)-L\left(\hat{\rho}_{k}\right) C
\end{array}\right]\left(\begin{array}{c}
x_{k} \\
e_{k}
\end{array}\right)
$$

Theorem 11: Let an observer (8) that satisfies the hypotheses of Theorem 4 and a state feedback law that satisfies the hypotheses of Theorem 8 be given ${ }^{2}$. Then for any $\max \left\{1-\frac{1}{\sigma_{e v}}, 1-\frac{1}{\sigma_{x v}}\right\} \leq \varepsilon<1$ and any $0<\beta \leq \frac{1-(1-\varepsilon) \sigma_{e v}}{\mu^{-2} \sigma_{x e}}$ the closed-loop system (24) is GES with decay factor equal to $\sqrt{\varepsilon}$ for all uncertainties satisfying $\|\Delta A(\rho, \hat{\rho})\| \leq \delta:=$ $\sqrt{\frac{\beta\left(1-(1-\varepsilon) \sigma_{x v}\right)}{\sigma_{e v}+\beta \sigma_{x v} v}}$.

Proof: See [16] for the proof.

It is of interest to find the value of $\beta$ that provides the largest robustness in terms of $\delta$. To maximize the value for $\delta^{2}$ (for a fixed value of the decay factor $\sqrt{\varepsilon}$ ) it is clear that we have to maximize $f(\beta):=\frac{\beta}{\sigma_{e v}+\beta \sigma_{x v}}$. Since $\frac{d f(\beta)}{d \beta}=\frac{\sigma_{e v}}{\left(\sigma_{e v}+\beta \sigma_{x v}\right)^{2}} \geq 0$, the maximum is obtained for the largest allowable value of $\beta$, which is $\frac{1-(1-\varepsilon) \sigma_{e v}}{\mu^{-2} \sigma_{x e}}$ and thus the maximum of $\delta$ is

$$
\delta(\varepsilon)=\sqrt{\frac{\left(1-[1-\varepsilon] \sigma_{e v}\right)\left(1-[1-\varepsilon] \sigma_{x v}\right)}{\mu^{-2} \sigma_{x e} \sigma_{e v}+\left(1-[1-\varepsilon] \sigma_{e v}\right) \sigma_{x v}}} .
$$

Suppose we now would like to find the value of $\varepsilon$ such that the admissible uncertainty level $\delta(\varepsilon)$ is maximal. Since $\frac{d \delta^{2}(\varepsilon)}{d \varepsilon}=\frac{\left[1-(1-\varepsilon) \sigma_{e v}\right] \sigma_{x v}}{\mu^{-2} \sigma_{x e} \sigma_{e v}+\left[1-(1-\varepsilon) \sigma_{e v}\right] \sigma_{x v}} \geq 0$ for any $\max \{1-$ $\left.\frac{1}{\sigma_{e v}}, 1-\frac{1}{\sigma_{x v}}\right\} \leq \varepsilon<1$, maximizing robustness requires maximizing (actually taking supremum of) $\varepsilon$ and thus taking it close to 1 . This yields that the maximal value of $\delta$ can become arbitrarily close to

$$
\delta(1)=\sqrt{\frac{1}{\mu^{-2} \sigma_{x e} \sigma_{e v}+\sigma_{x v}}}
$$

while still guaranteeing stability. Hence, for maximizing robustness in terms of maximizing $\delta(\varepsilon)$, we should maximize $\varepsilon$ meaning that the performance in terms of the decay factor $\sqrt{\varepsilon}$ is worst. As such, we encountered a "classical" tradeoff between robustness and performance.

The reasoning above maximizes robustness for fixed values of $\sigma_{x v}, \sigma_{e v}$ and $\sigma_{x e}$. Since we have determined this maximum given these $\sigma$ 's, we can now optimize robustness by appropriately selecting the gains $L_{i}$ and $K_{i}, i=1, \ldots, N$. From (26) it is clear that we have to minimize $\mu^{-2} \sigma_{x e} \sigma_{e v}+$ $\sigma_{x v}$ to get the maximal value for $\delta(1)=\sqrt{\frac{1}{\mu^{-2} \sigma_{x e} \sigma_{e v}+\sigma_{x v}}}$ (for decay factor equal to 1 ). This gives rise to the following procedure to get maximal robustness in the mismatch between the scheduling parameter $\hat{\rho}_{k}$ and the actual one $\rho_{k}$ as reflected in $\delta$.

\section{Design procedure}

Step 1: Minimize $\sigma_{e v}$ subject to (11) for $i, j=1, \ldots, N$ (i.e. optimization problem (14)). This gives the minimum $\sigma_{e v}^{*}$ and the corresponding observer gains $L_{i}, i=1, \ldots, N$.

Step 2: Given $\sigma_{e v}^{*}$ at in Step 1. Fix $\mu>0$ and minimize the expression $\mu^{-2} \sigma_{x e} \sigma_{e v}^{*}+\sigma_{x v}$ subject to the

\footnotetext{
${ }^{2}$ Note that the hypotheses guarantee that $\sigma_{e v}>1$ and $\sigma_{x v}>1$.
}

LMIs given in (18). This results in the feedback gains $K_{i}, i=1, \ldots, N$.

The optimization problems in Step 1 and 2 are convex problems as we are minimizing linear costs subject to LMI constraints. Step 2 might even be extended by performing a line search in $\mu$ and applying the above procedure repetitively. Once, the minimal value $\mu^{*-2} \sigma_{x e}^{*} \sigma_{e v}^{*}+\sigma_{x v}^{*}$ is found, one can on the basis of Theorem 11 and (25) still make tradeoffs between transient performance in terms of the decay factor $\sqrt{\varepsilon}$ and robustness in terms of $\delta(\varepsilon)$. Letting $\varepsilon$ increase from $\max \left\{1-\frac{1}{\sigma_{e v}}, 1-\frac{1}{\sigma_{x v}}\right\}$ (maximal performance, minimal robustness) to 1 (minimal performance, maximal robustness), tradeoff curves between performance and robustness are obtained.

\section{ILLUSTRATIVE EXAMPLE}

Consider the LPV system (5) with $A\left(\rho_{k}\right)=$
$\left[\begin{array}{ccc}0.25 & 1 & 0 \\ 0 & 0.1 & 0 \\ 0 & 0 & 0.6+\rho_{k}\end{array}\right], B=\left[\begin{array}{lll}1 & 0 & 1\end{array}\right]^{T}, C=\left[\begin{array}{lll}1 & 0 & 2\end{array}\right]$,
$D=0$ and $\rho_{k} \in[0,0.5], k \in \mathbb{N}$. In this case we can take the functions $\xi^{1}(\rho)=\frac{0.5-\rho}{0.5}$ and $\xi^{2}(\rho)=\frac{\rho}{0.5}$ with $A_{1}=A(0)$ and $A_{2}=A(0.5)$. The observer is designed using Theorem 2 along with the optimization problem (14) (Step 1). The optimal solution is given by $\sigma_{e v}^{*}=5.8277$ with observer gains $L_{1}=\left[\begin{array}{lll}-0.0835 & -0.0011 & 0.3870\end{array}\right]^{T}$ and $L_{2}=\left[\begin{array}{lll}-0.0835 & -0.0011 & 0.7094\end{array}\right]^{T}$. With this optimal observer and the associated slope of the linear ISS gain $\sigma_{e v}^{*}$, a line search involving $\mu>0$ is performed in order to minimize the cost $J=\mu^{-2} \sigma_{x e} \sigma_{e v}^{*}+\sigma_{x v}$ subject to the LMIs given in (18) for all $i, j$ (Step 2). Fig. 1 shows the minimum of $J$ for each fixed $\mu$, which is the smallest Note that for each fixed $\mu$ we have a convex programming problem to solve (linear cost criterion with LMI constraints). for $\mu^{*}=0.2986$ yielding $\sigma_{x e}^{*}=0.2663$ and $\sigma_{x v}^{*}=13.9284$ and corresponds to the controller gains $K_{1}=\left[\begin{array}{lll}-0.0327 & -0.1241 & -0.2387\end{array}\right]$, $K_{2}=\left[\begin{array}{lll}0.0005 & -0.0010-0.6148\end{array}\right]$.

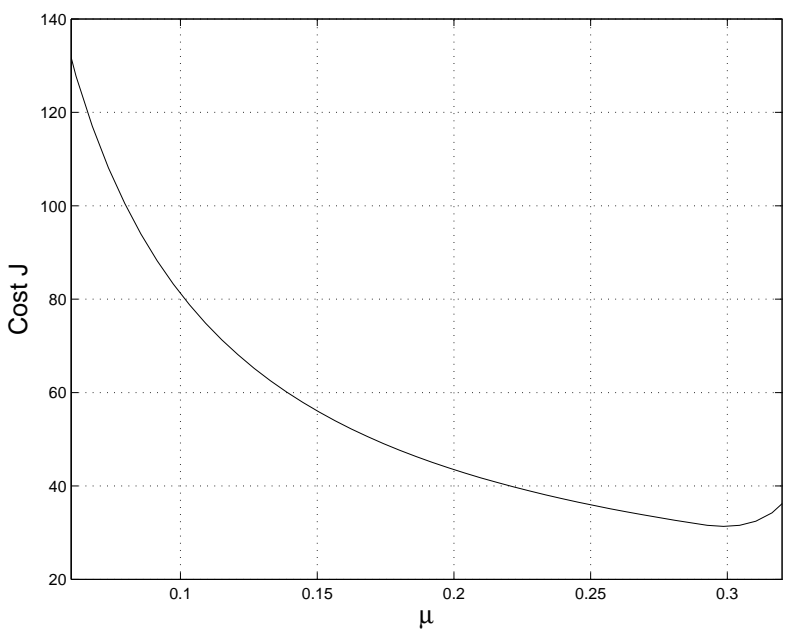

Fig. 1. Line search $\mu$ 


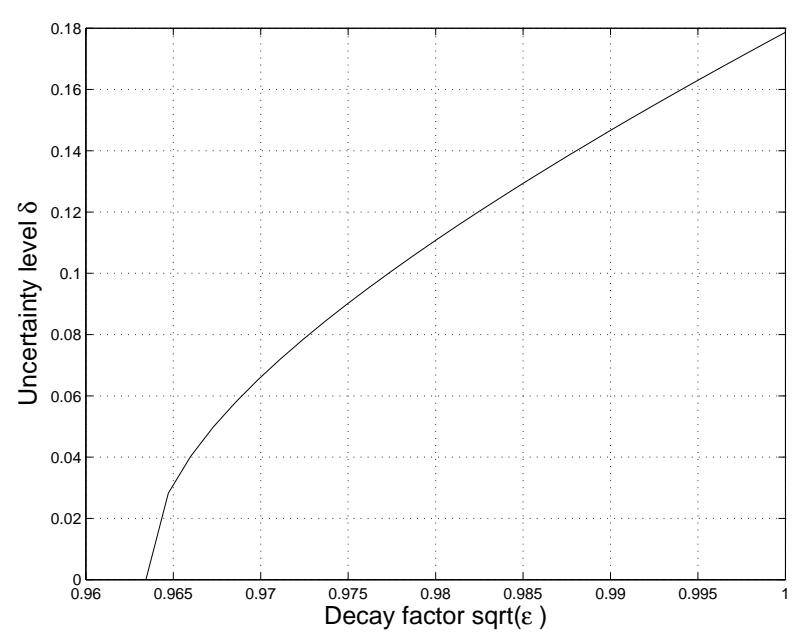

Fig. 2. Tradeoff performance/robustness

As a consequence, the maximum level of uncertainty is $\delta_{\text {max }}^{*}=\sqrt{\frac{1}{\mu^{*-2} \sigma_{x e}^{*} \sigma_{e v}^{*}+\sigma_{x v}^{*}}}=0.1786$. Hence, for $\Delta A\left(\rho_{k}, \hat{\rho}_{k}\right)=\left|\rho_{k}-\hat{\rho}_{k}\right| \leq \delta<0.1786$ GES of the closedloop system (24) is guaranteed (with a decay factor close to 1). Letting $\varepsilon$ increase from $\max \left\{1-\frac{1}{\sigma_{v v}^{*}}, 1-\frac{1}{\sigma_{v v}^{*}}\right\}$ to 1 leads to the tradeoff curves between performance in terms of the decay factor $\sqrt{\varepsilon}$ and robustness to uncertainty $\Delta A\left(\rho_{k}, \hat{\rho}_{k}\right)$ in terms of $\delta$ as depicted in Fig. 2.

\section{CONCLUSIONS}

In this paper the design of robustly stabilizing outputbased feedback controllers is considered for discrete-time LPV systems in which the scheduling parameters are only known up to a given precision. The output-based controllers are obtained using separate design of the observer and the state feedback and we showed that the interconnection of the LPV plant, observer and state feedback leads to a stable closed-loop system for certain levels of mismatch between estimated and true parameters. The non-conservatism of our approach is demonstrated by showing that well known conditions for nominally stabilizing observers and feedbacks (i.e. without mismatch between true and available parameters) imply our LMI-based conditions. The flexibility in the framework allows to construct the controller that guarantees stability for the largest level of parameter uncertainty and to make tradeoffs between performance in terms of decay factor and robustness with respect to parameter uncertainty.

\section{REFERENCES}

[1] J. Shamma and M. Athans. Guaranteed properties of gain scheduled control of linear parameter-varying plants. Automatica, 27:559-564, 1991.

[2] G. Becker and A. Packard. Robust performance of linear parametrically varying systems using parametrically-dependent linear feedback. Syst. Contr. Lett., 23:205-215, 1994.

[3] P. Apkarian and P. Gahinet. A convex characterization of gainscheduled Hinfinity controllers. IEEE Trans. on Automatic Control, 40:853-864, 1995.

[4] W.J. Rugh and J.S. Shamma. A survey of research on gain-scheduling. Automatica, 36:1401-1425, 2000.
[5] C. Scherer. LPV control and full block multipliers. Automatica, $37: 361-375,2001$.

[6] F. Blanchini and S. Miani. Stabilization of LPV systems: state feedback, state estimation, and duality. SIAM J. Control Optim., 42:7697, 2003.

[7] I.E. Kose and F. Jabbari. Control of LPV systems with partly-measured parameters. Proceedings of the 36th IEEE Conference on Decision and Control, 1997.

[8] J. Daafouz, J. Bernussou, and J.C. Geromel. On inexact LPV control design of continuous-time polytopic systems. IEEE Trans. Automatic Control, 53:1674-1678, 2008.

[9] G. Millerioux, L. Rosier, G. Bloch, and J. Daafouz. Bounded state reconstruction error for LPV systems with estimated parameters. IEEE Trans. Automatic Control, pages 1385-1389, 2004.

[10] E. D. Sontag. Smooth stabilization implies coprime factorization IEEE Trans. Automatic Control, 34:435-443, 1989.

[11] Z.-P. Jiang and Y. Wang. Input-to-state stability for discrete-time nonlinear systems. Automatica, 37:857-869, 2001.

[12] F. Blanchini, S. Miani, and F. Mesquine. A separation principle for linear switching systems and parametrization of all stabilizing controllers. Proc. IEEE Conference on Decision and Control, pages 953-958, 2008.

[13] A. Alessandri, M. Baglietto, and G. Battistelli. Luenberger observers for switching discrete-time linear systems. Int. J. Control, 80(12):1931-1943, 2007.

[14] S. Boyd, L. El Ghaoui, E. Feron, and V. Balakrishnan. Linear Matrix Inequalities in System and Control Theory, volume 15. SIAM Studies in Applied Mathematics, 1994.

[15] M. Lazar, D. Munoz de la Pena, W.P.M.H. Heemels, and T. Alamo. On the stability of min-max nonlinear model predictive control. Syst. Contr. Lett., 57(1):39-48, 2008.

[16] W.P.M.H. Heemels, J. Daafouz, and G. Millerioux. Observer-based control design for discrete-time LPV systems with unknown parameters. Submitted for journal publication.

[17] A.Lj. Juloski, W.P.M.H. Heemels, and S. Weiland. Observer design for a class of piecewise linear systems. Intern. J. Robust and Nonlinear Control, 17(15):1387-1404, 2007. 